\title{
Model of Technological, Managerial and Marketing Infrastructure for Intelligent Technology Efficiency in Telecommunication Industry - Case Study: Telecommunication Infrastructure Company of llam Province ${ }^{1}$
}

\author{
Heshmat Moradhaseli \\ scholar, Information Technology management college, Islamic azad university, central Tehran branch, Tehran, iran \\ h.haseli123@gmail.com \\ Jalal Haghighat Monfared* \\ Assistant Professor, Faculty of Management, Islamic Azad University, Central Tehran Branch, Tehran, Iran \\ fhmfhm3211@gmail.com
}

Received: 01/Jan/2020

Revised: 04/Feb/2020

Accepted: 28/ Apr /2020

\begin{abstract}
Today's, intelligent agent system (IAS) are considered as an important part of people's lives. Therefore, many of organizations try to implement IAS in their mechanism. One of these organizations in Iran is telecommunication Infrastructure Company. Because any implementation need a model which clarify the structural and contextual components, therefore, the current research is conducted to provide a model for developing the necessary infrastructure for implementation of intelligent technologies in the communication and telecommunication mechanisms of Ilam Province. To achieve the goal, a qualitative approach and thematic analysis method were used. The research population consisted of all experts in the field of ICT in Ilam province Infrastructure Communications Company that using purposeful sampling method and relying on theoretical data saturation, 10 of them were selected as sample. Semi-structured interviews were used to collect the data. The data were analyzed through theme analysis. Based on the method, 4 themes, 10 main categories and 153 open codes were extracted. The findings of the study showed that to transform communication mechanisms into intelligent technologies, there must be technological, management, marketing and cultural infrastructure. Technological infrastructure consisted of intelligent software and hardware; management infrastructure consisted of knowledge and belief; marketing infrastructure included attracting intelligent technology to audiences, encouraging ideas, physical and virtual channels; and finally, cultural infrastructure, it was staff training and public awareness.
\end{abstract}

Keywords: Intelligent Agent System; Intelligent Technology; Intelligent Communication; Technological Infrastructure; Management Infrastructure; Marketing Infrastructure.

\section{1- Introduction}

The industrial revolution experience and the age of enlightenment in many countries have led to the everexpanding and accelerating movement of societies towards growth and development, and the required tools and technologies were produced and offered to facilitate this process. However, technologies have played a significant role in these societies, and new generations of these technologies are increasingly being introduced today, but intelligent technologies has been conducted in the different area. Therefore, the need for the intelligent technology is very urgent.

In the past, communication was thought to be merely a one-way process in which the information holder transmits information to its recipients [1] as there were only one or more suppliers presenting their information. But with the advent of technologies, communication mechanisms have changed dramatically. On the other hand, not only macrotechnology policies have been based on the equipment of existing technologies with intelligent technologies, but there has also been a widespread social wave in society that calls for intelligent acting of their social and organizational relationships [2], [3]. Now, if this tendency is to take an organized form and to apply such an approach to the country's telecommunications system, there needs to be a coherent conceptual model to build the necessary infrastructure on it.

Today, telecommunications systems have complex tasks. It is not only expected that traditional communication tools are truly established in every country, but new generations of information technologies, especially intelligent technologies, are also expected to grow. Meanwhile,

\footnotetext{
* Corresponding Author

1. Extracted article of Phd.Thesis
} 
connectionism has emerged as a significant paradigm in the telecommunications industry. Under this paradigm, telecommunications companies strive to serve their customers through its connections. In total, there are two orientations in connectivism theory. One is the orientation of Stephen Downes, who works in the field of online communication content composition, and the other is George Siemens's approach [4, 5]. Downs focuses more on connective knowledge and communication and considers it an interactive knowledge that takes place on a social network. But Siemens sees connectivism as more of a communication paradigm for the digital age [5].

From a connectionism standpoint, we acquire our knowledge, interactions, relationships, and learnings through the connections we make or the connections we belong to. Downes defines connectivism as a communication theory that considers knowledge as the creation of a network of interactions [4]. In fact, networks are created from a set of interactions between professionals, students, professors, general public, databases, blogs, colleges, telecommunications companies and websites, and learning in such an environment requires the ability to create and develop networks [6]. However, the basic theory of this research is based on connectionism. This theory is based on the following dimensions and components:

1. Connectionism interactions are based on diversity of opinions and behaviors [7].

2. Learning and communication is the process of connecting to nodes and information resources [8].

3. Communications may be created through non-human (machine) applications [9].

4. Communication capacity and ability in how to communicate is much more important than communication itself [10].

5. Maintaining and developing continuous communication is essential [11].

6. The ability to understand the connections between branches, thoughts and concepts is an essential skill [12].

7. Up-to-date and accurate knowledge is the main goal of all connectionism communication activities [13].

8. Decision making itself is a kind of learning. Choosing what to learn and the meaning of the input information is itself a kind of learning [14].

On the other hand, by looking at these dimensions, it can be seen that in order to establish a connectionism intelligent system, it is necessary to provide the appropriate infrastructure. Daft [16] believes that these infrastructures which are needed to establish information technology includes three infrastructures: technological infrastructure, management infrastructure, and marketing infrastructure. Therefore the present study considered these foundations and infrastructures as the research foundations.

The research questions are:
1. What kind of technological infrastructures are necessary for the development of intelligent technologies in the Infrastructure Communications of Ilam Province?

1. What kind of managerial infrastructures are necessary for the development of intelligent technologies in the Infrastructure Communications of Ilam Province?

1. What kind of marketing infrastructures are necessary for the development of intelligent technologies in the Infrastructure Communications of Ilam Province?

The present study aimed to provide a clear answer to these questions. The purpose of this study is to identify the areas and infrastructure needed for the development of intelligent technologies in Telecommunication Infrastructure Company of Ilam. In addition, the research was seeking to address the contexts, infrastructures, and backgrounds should be provided to transform the Telecommunication Infrastructure Company's mechanisms into intelligent communications.

Ignoring this model to develop intelligent technologies can lead to high costs for the organization. Because the use of traditional generations of information technology always requires the existence of significant agents and human resources to exploit it. But intelligent technologies conducted their job intelligently without human intervention and without the need for constant monitoring. On the other hand, ignoring intelligent technologies deprives the infrastructure communications company of the ability to respond quickly and efficiently. Because today, one of the indicators that is considered by the audience and customers of telecommunication and communication companies is the ability to provide easy, fast and quality service, all of which can be achieved by intelligent technologies.

\section{2- Theoretical Background}

Intelligent systems or intelligent agent systems have different types. However, the common feature of all these systems is that they can carry out their activities without human intervention. These systems also include very simple and basic systems such as thermostats they also include more sophisticated systems, such as simulation systems or learning management systems. Some intelligent systems have the ability to learn in new situations and others can only adapt to different situations. Intelligent systems must have the capacity to recognize the status quo and make appropriate decisions and actions for different situations.

There are many different types of intelligent systems, but all of them have four features in common which are:

1. Independence and self-control

2. Activity

3. Accountability

4. Adaptability [5]. 
One of the features of intelligent systems is their flexibility and adaptability. In the context of communication, such a feature means identifying the needs of users and providing the necessary feedback and information resources for them. Existing systems focus more on a particular aspect of communication. In the world of communication and education, part of intelligent systems are intelligent cognitive systems that are responsible for recognizing users' cognitive level and responding appropriately to their cognitive needs. Part of it focuses on the emotional aspects of users and provides appropriate responses based on the emotional state of users. In some researches, showed that using several strategies, intelligent systems can be built based on Web 2 and the linking system. These strategies are providing credible knowledge, management integration management guide, data recovery on time, provide data in a homogeneous manner [5]. It should be noted that the effect of increased agent transparency on operator workload depends on the amount of information provided and whether the information is required for the operator's task performance [6]. This is while it has been stated before that intelligent systems perform their activities without the need for human intervention. In its absence of a user manual, an intelligent entity must learn to align its values with that of humans [7]. It is in such a complex world that intelligent systems are introduced [8]. However, some believe that the deployment of intelligent systems requires different infrastructures [9]. Among these, they have shown that technological, cultural, marketing and management infrastructures are among the most important infrastructures [10].

\section{3- Related Works}

Mohammadi 2017 designed a conceptual model of intelligent learning management system responsive to cultural diversity of Mehr Alborz virtual university students. This research was conducted using a mixed methods and its findings showed that to govern a multicultural approach in our higher education system, its infrastructure that include multicultural education policy and multicultural human resources are needed to be provided first to respond to the diverse needs of cultural minority students by relying on macro education strategies (at university and ministry level) and micro strategies (at classroom level). Based on the findings of the first part of the research, the conceptual model of the intelligent system was also designed and validated, whereby the present systems, curriculum content, educational activities and specific cultural programs were presented to each student. Finally, suggestions were made to deploy the system in question [3].

In another study, Rocher, Janse, Portolan and Streitz (2014) examined the demands of users of intelligent home environment systems. Ambient Intelligence encompasses the concept of future life, with much of the day-to-day home activities being carried out by intelligent systems. The study sought to identify and categorize the demands that different users of various cultures from the system. To achieve this goal, mixed research method was used. The findings of the study showed that user expectations and demands for the intelligent home environment system are as follows: 1 . The system can detect all user behaviors and never go astray; 2 . The system must be highly secure and protected to be able to provide proper privacy for users; 3 . The system should be able to gain added value compared to other existing systems; 4 . The system should never replace actual interactions between people in unnecessary situations; 5. Convenience and comfort should always be preserved at home and the system should not disturb it [15]. Mazadi, Qasim Aghaei and Oren, (2008) in another study merely sought to model culture and its dimensions for simulation in culturally-intelligent systems. The findings of this study showed that the design of this system required the following information to be collected: 1 . Identifying the nationality of each user, since Schwartz's research provides comprehensive information about the cultural characteristics of each nation and can be used to identify the cultural characteristics of each user. 2 . Learning from the behaviors and performance of the student (user) according to which the cultural characteristics of the user can be classified based on the indicators specified in different cultural categories. 3 . Assessment of student's cultural characteristics through PVQ questionnaire [16].

\section{4- Research Methodology}

To achieve the purpose of the present study, qualitative approach and theme analysis were used. The qualitative approach was used because the pattern of intelligent technologies had to be consistent with the cultural, political, economic, and social context of any society, and identifying such a context would probably require qualitative research. On the other hand, identifying the dimensions and components needed to transform existing communication into intelligent communication requires an indigenous model appropriate to the cultural, social and economic context of the community in question. For this reason, the theme analysis research method can be instrumental in achieving this goal. Theme analysis attempts to extract themes and content from various discourses, interviews, and texts, and to identify the focal ideas of those documents [15].

The research population consisted of all experts in the field of ICT in Telecommunication Infrastructure Company of Ilam. Of these, 10 were selected using purposive sampling. In addition, sample size estimates were based on 
theoretical data saturation. It was purposeful because the researcher consciously, not based on randomize selection, selects people who have specialized knowledge and attitudes about the phenomenon and can provide in-depth information to the researcher. The reason for the selection of these people was that they all had educational backgrounds and academic knowledge in the field of information technology, especially intelligent technologies. Secondly, all of them were operating and managing the information systems operating and operating in the Telecommunication Infrastructure Company of Ilam. Thus, all interviewees had adequate theoretical and conceptual knowledge of intelligent technologies.

The data collection tool was semi-structured interview. The reason for using this type of interview was to allow the researcher to analyze and evaluate their perspectives and experiences without limiting the participants' views to a particular context or basis.

1. In your opinion, what kind of technologies features should be able to run and implement intelligent systems in order to make intelligent communication technology in Ilam? 2. In your opinion, what kind of management and leadership approaches should be able to run and implement intelligent systems in order to make intelligent communication technology in Ilam?

3. what kind of marketing procedure should be able to run and implement intelligent systems in order to make intelligent communication technology in Ilam?

Finally, the researcher analyzed and interpreted the research findings using a thematic analysis method based on two types of open coding and axial coding. In this type of analysis, information is first collected from the participants and then the data is coded, without any specific theoretical basis (open coding). Next, duplicate and redundant codes are removed, and the process of code reductions continues until we reach broader categories that can include more minor codes called axial coding [17]. During the open coding procedure, 153 open codes were extracted. After the open coding procedure, a number of duplicate and redundant codes were removed and 10 main categories and 4 general themes were extracted by constant reference to interviews, analysis, comparison and review of data. What is presented in the following table is a report of extracted sample codes, subcategories, main categories and main research theme.

Two methods were used to validate the research data. First, Triangulation which is the process of corroborating evidence from different individuals (e.g., a principal and a student), types of data (e.g., observational field notes and interviews), or methods of data collection (e.g., documents and interviews) in descriptions and themes in qualitative research. Second, Member checking is a process that the researcher asks one or more participants in the study to check the accuracy of the account.

\section{5- Data and Findings}

In view of the tangible relationship between the experts of the Telecommunication Infrastructure Company with the research problem, they were asked to participate in the present study and to provide their views on the research questions. What follows are the findings from the interviews with these individuals, which are summarized and analyzed in Table 1.

Table 1. Themes and categories extracted from the interviews

\begin{tabular}{|c|c|c|}
\hline Themes & Main categories & extracted sample codes \\
\hline $\begin{array}{l}\text { Technological } \\
\text { infrastructure in the } \\
\text { telecommunications } \\
\text { industry }\end{array}$ & $\begin{array}{l}\text { - Intelligent } \\
\text { hardware } \\
\text { - Intelligent } \\
\text { software }\end{array}$ & $\begin{array}{l}\text { Purchasing the right equipment; } \\
\text { Purchasing the right software; } \\
\text { Sufficient bandwidth; IT based } \\
\text { communication channels; } \\
\text { Providing telecommunication } \\
\text { data such as fiber, FTTH, ADS; } \\
\text { high speed Internet. }\end{array}$ \\
\hline $\begin{array}{l}\text { Managerial } \\
\text { infrastructure in the } \\
\text { telecommunications } \\
\text { industry }\end{array}$ & - Knowledge & $\begin{array}{c}\text { Targeted management; } \\
\text { Committed to adherence to goals } \\
\text { and methods; Scientific } \\
\text { evaluation of programs and } \\
\text { goals; Defining intelligent } \\
\text { strategic perspectives; Defining } \\
\text { medium-term and long-term } \\
\text { goals, mechanisms and } \\
\text { structures; } \\
\text { The firm conviction that } \\
\text { inteligent ing is performed; } \\
\text { Manager awareness of intelligent } \\
\text { features; manager awareness of } \\
\text { intelligent services and benefits }\end{array}$ \\
\hline $\begin{array}{l}\text { Marketing } \\
\text { infrastructure in the } \\
\text { telecommunications } \\
\text { industry }\end{array}$ & $\begin{array}{c}\text { - Attracting } \\
\text { intelligent } \\
\text { technologies } \\
\text { - Encouraging } \\
\text { people with ideas }\end{array}$ & $\begin{array}{l}\text { Proving intelligent Technology } \\
\text { Attraction for Investor } \\
\text { Companies, Providing } \\
\text { Reliability to Investor } \\
\text { Companies for Investing, } \\
\text { Providing Return on Investment } \\
\text { for Investor Companies }\end{array}$ \\
\hline $\begin{array}{l}\text { Cultural } \\
\text { infrastructure in the } \\
\text { telecommunications } \\
\text { industry }\end{array}$ & $\begin{array}{l} \\
\text {-Staff training } \\
\text { - Informing } \\
\text { people } \\
\text { Virtual channels }\end{array}$ & $\begin{array}{c}\text { Raising public awareness, use of } \\
\text { visual media for awareness, use } \\
\text { of print media for awareness, use } \\
\text { of national media for awareness, } \\
\text { use of virtual spaces for } \\
\text { awareness, full awareness of } \\
\text { intelligent features to Client, } \\
\text { Human Resources Training }\end{array}$ \\
\hline
\end{tabular}

The findings of this study revealed that all of these infrastructures can be summarized and categorized into four infrastructures. What follows is a report of findings from experts in this field.

\section{A) Technological Infrastructure in the Telecommunication Industry}

Findings from the analysis of the interviews showed that to become an intelligent organization and to make Ilam's telecommunications mechanisms intelligent, it is necessary to develop and strengthen existing technologies. This development can be examined in two dimensions. 


\section{Hardware Development}

Interviewees believe that in order to integrate intelligent technologies into communication mechanisms, existing hardware needs to be further developed. They believe that in order to become more sophisticated, communication infrastructure needs to have specific hardware in place. For example, interviewee No. 4 has raised important points in this regard.

"To become intelligent, you have to acquire good hardware infrastructure. We need wider bandwidth. Otherwise, many intelligent programs won't run at all."

\section{Software Development}

Interviewees not only believe that the company's existing hardware needs to be upgraded, but some interviewees also believe that the existing software infrastructure is inadequate and needs to be strengthened. In this regard, the viewpoint of interviewee No. 3 can be considered.

"In order to be able to implement intelligence, we need to strengthen the software of the Telecommunication Infrastructure Company. We need to have the telecommunications data such as fiber and ADSL to be optimized so that it can be used for intelligent purposes."

\section{B) Management Infrastructure in the Telecommunications Industry}

In order to find intelligent technologies in any organization, it is not only necessary to provide the right software and hardware infrastructures, but also to efficiently manage the organization. Based on the data obtained from the interviews, the management infrastructure can be examined in the following two dimensions.

\section{Knowledge of Managers}

Interviewees strongly believe that a manager who does not have the appropriate knowledge and information required by intelligent technologies cannot provide the appropriate context for its advancement. To this end, interviewee No. 4 has put forward such a view.

"In my opinion, a manager who wants to implement intelligent technologies must first have sufficient knowledge and expertise about these technologies. A specialized manager can provide the necessary background for the success of intelligent technologies."

\section{Managers' Beliefs}

Data from interviews with IT professionals suggest that there must be a firm belief that these technologies are effective and efficient. Some of the participants such as interviewee \# 1 are serious critics of existing sloganistic approaches and believe that only by relying on the true belief of managers can these technologies be successfully implemented.
"Organizers must have a firm belief in intelligent technologies, not in slogans or in luxury, performing no appropriate action." "

\section{C) Marketing Infrastructure in the Telecommunications Industry}

Today's world is accompanied with increasing competition for organizations and companies to achieve sustainable development. In this regard, outsourcing and privatization is one of the most important steps that organizations take to succeed in their intelligent mechanisms. The marketing infrastructure can be discussed in the following dimensions.

\section{Make Intelligent Technologies Attractive}

Data from interviews with experts suggest that in order to attract customers for intelligent technologies, they must be offered as an attractive technology. Some interviewees have explicitly stated that in order to be successful in privatizing and outsourcing intelligent technologies, the necessities and benefits of inteligent ening must be clearly stated for outsider companies. For example, interviewee No. 5 has commented on this.

"To be successful in this (intelligent marketing) business, one has to realize the benefits of using intelligent technologies for investor companies."

\section{Encourage the Proponents}

Many interviewees believe that in order to be successful in marketing intelligent technologies, it is important to encourage those who have ideas to invest in intelligent technologies or implement their technological ideas. Various approaches have been suggested by the interviewees, but overall, many believe that telecommunications companies should be able to provide the opportunity to attract creative people. Interviewee No. 5 expressed his view in this regard.

"Proponents should be encouraged to invest in intelligent technologies or implement their ideas."

\section{Advertise Via Virtual Channels}

With the advent of virtual communications, today, it is expected that intelligent technology mechanisms will also be propagated through virtual channels. Some interviewees believe that these technologies can be advertised using the infrastructure systems and automation. Interviewee No. 8 has identified the Enterprise Portal of the Telecommunication Infrastructure Company as an important channel for advertising.

"The Telecommunication Infrastructure Company's Portal itself is an important channel for outreach. Because anyone who visits an organization's website will see the most important news and announcements and can take action in investment." 


\section{D) Cultural Infrastructure of the Telecommunications Industry}

Intelligent human activities also require cultural infrastructure to enable users of both technologies to learn how to use those technologies and to minimize the potential for misuse of these technologies. Interviewees believe that cultural infrastructure is a key pillar for inteligent ening, and without it we cannot hope for the successful implementation of intelligent technologies. In sum, cultural infrastructure can be studied in two dimensions, each of which is discussed below.

\section{Staff Training}

Interviewees believe that effective and efficient cultures for intelligent technologies are possible only if the providers of those technologies have the appropriate knowledge, recognition, and attitude of those technologies. Interviewees have abundantly stated that providers of these technologies must have a precise and accurate understanding of intelligent technologies in order to effectively train and cultivate.

For example, interviewee No. 6 reflected this belief in his statement.

"Adequate training should be given to staff on how to use intelligent technologies."

\section{Informing People}

The end users of intelligent technologies include the people who are always the clients of these technologies. In order to realize all the positive benefits of intelligent technologies, people need to be aware of the mechanisms and how to use them. Interviewees believe that informing people is an essential step for cultural contexts. Interviewee \# 7 identified informing people as a key step in cultivation.

"People need to be informed on how to use intelligent technologies. As long as information is not available, the benefits of intelligent technologies cannot be effectively exploited."

\section{5-1- System Model}

Nevertheless, a review of the findings of this study revealed that in order for intelligent technologies to be well developed, all four infrastructures needed to be interoperable. On the other hand, these findings suggest that all four infrastructures play an essential and effective role in the implementation of intelligent technologies. The final model of the research is shown in Figure 1.

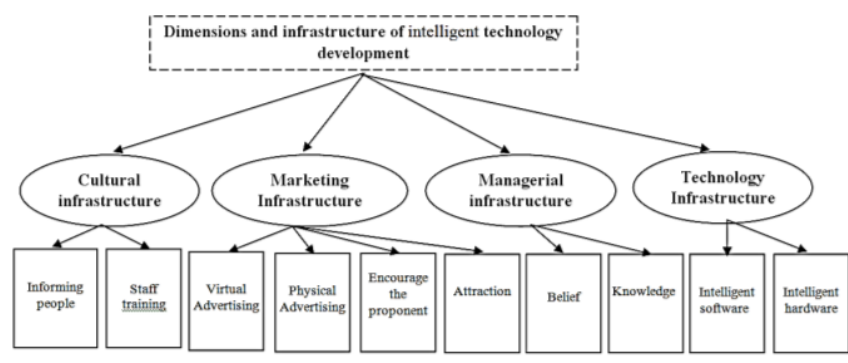

Fig. 1. Final research model

\section{6- Results and Discussion}

The findings of this study showed that in the first place, to transform existing communications into intelligent communications, there must be adequate hardware and software infrastructure in the company. Purchasing the right equipment; Purchasing the right software; Sufficient bandwidth; IT-based communication channels; Providing telecommunication data such as fiber, ADSL; FTTH; all were suggestions made by the participants. This part of the research findings is consistent with the findings of Mohammadi 2017, Rocher and et al 2014 and Mazadi and et al 2008 researches. Because they have also shown that there must be technological infrastructure for intelligent technologies.

Second, the company's management needs to be flexible and, in this respect, the most central emphasis is on executives who are responsible for implementing intelligent systems. Interviewees believed that relevant managers should have both an appropriate knowledge and information about intelligent technologies and a strong belief in the effectiveness of these technologies. As such, the firm conviction about intelligent making; manager awareness of the intelligent making features; and manager awareness of the services and benefits of intelligence were among the suggestions the interviewees made. This part of the findings is new. Because previous studies have not been addressed this issue.

Third, marketing infrastructure is also one of the most important infrastructures needed for intelligent communication and telecommunications activities. In the meantime, the findings of the study showed that to provide such an infrastructure, intelligent technologies must be attractive to investor companies, encourage proponents to invest in the field, and eventually take place through appropriate and effective virtual advertising channels. This part of the findings is new. Because previous studies have not been addressed this issue.

Fourth, there is a need for cultural infrastructure to transform communication mechanisms into intelligent mechanisms, and to provide such infrastructure, firstly, the staff of the communication infrastructure company must receive the necessary training and secondly, to provide 
appropriate information to the general public to implement the necessary cultivation.

This part of the research findings is consistent with the findings of Mohammadi 2017, Rocher and et al 2014 and Mazadi and et al 2008 researches. They all dealt with culture as a fundamental element

The present study includes innovation in several ways.

First, this research contributes to the development of literature because no comprehensive and serious research has been done on this issue in Iran so far, and the model presents new views to the intelligent world.

Second, the present research can help the planners and policy makers of the Infrastructure Communications Company to make coherent and codified plans to improve the state of intelligent technologies by relying on the data and evidence obtained from this research.

Third, this research has shown that in order to establish an intelligent system, not only technological infrastructure is needed, but also other infrastructures must be provided.

Based on the results, the following suggestions are made. 1. Since the present study showed that there must be appropriate technological infrastructure for the establishment of an intelligent system, it is recommended that the communications company provide and deploy the appropriate software and hardware infrastructure.

2. Since the findings showed that leadership and management skills are essential elements for intelligent technologies, it is recommended that appropriate training courses be held for current managers on the necessities of intelligent systems in organization.

3. Since the findings show that proper marketing should be done for intelligent technologies, it is recommended that the infrastructure communications company use virtual and physical methods to advertise its intelligent technologies.

\section{References}

[1]. Constantinides, E and Fountain, S. Web 2: conceptual foundation and marketing issues. Journal of Direct, Data and Digital Marketing Practice. Vol. 9, No. 3, 2008, 231-244.

[2]. Mohammed, Ph, Mohan, P. The design and implementation of an encultured web- based intelligent tutoring system for computer science education. The IEEE international Conference on advanced learning technology. 2011, 501-505.

[3]. Mohammadi. Sh. designing the pattern of intelligent learning responding to Cultural diverse of students in Mehr University of alborz. Thesis of educational Management of Tehran University. 2017.

[4]. Ahrens, A; Wen, M, L; Huang, Y; Zascerinska, J and Bassus, O. A comparative study of the relationship between social dimension of web 2.0 technologies and E- learning: students view in German and Taiwan. Paper presented at the 3rd International Conference on Innovation and Entrepreneurship and its implications in Engineering and Business Education, The La Consolacion College Manila, Manila, Philippines, 2010. 16 - 18 November.

[5]. Trannsue, B, M. Connectivism and information literacy: moving from learning theory to pedagogical practice. Public Services Quarterly, 9:3, 2014, 185-195.

[6]. Mercado, J, M; Rupp, M, A; Barbet, D \& Procci, K. Intelligent Agent Transparency in Human-Agent Teaming for Multi-UxV Management. Vol. 58, No. 3, May 2016, pp. 401-415.

[7]. Kumar, V; Dixit, A; Rajshekar, R \& Javalgi, G. Research framework, strategies, and applications of intelligent agent technologies (IATs) in marketing. J. of the Acad. Mark. Sci. DOI 10.1007/s11747-015-0426-9. 2017.

[8]. Rosenfeld, A; Agmon, N; Maksimov, O; Azaria, A \& Kraus, S. Intelligent Agent Supporting Human-Multi-Robot Team Collaboration. Proceedings of the Twenty-Fourth International Joint Conference on Artificial Intelligence, 2015.

[9]. Lemoine, P, M, P; Trentesaux, D; Rey, G, Z \& Millot, P. Designing Intelligent Manufacturing Systems through Human-Machine Cooperation Principles: A Human-Centered Approach. Computers \& Industrial Engineering, Volume 111, September 2017, Pages 581-595.

[10]. Dermeval, D; Paiva, R; Borges, D; Bittencourt, I, I \& Vassileva, J. Authoring Tools for Designing Intelligent Tutoring Systems: A Systematic Review of the Literature. International Journal of Artificial Intelligence in Education volume 28, pages336-384, 2018.

[11]. Lin, Ch, F; Yeh, Y, Ch; Hung, Y, H and Chang, R, I. Developing an intelligent agent for managing web 2.0 services. International conference on future computer supported education. IEPRI, 2, 2012, 738-741.

[12]. Alexander, S, Th, V. An affect-sensitive intelligent tutoring system with an animated pedagogical agent that adapts to student emotion like a human tutor. A thesis presented at Massey University, Albany, New Zealand. 2007.

[13]. Anderson, T and Dron, J. Three generations of distance education pedagogy. International review of research in open and distance learning. 2011, Vol. 12.3.

[14]. Blanchard, E and Frasson, C. Making intelligent tutoring systems culturally aware: the use of Hofstede's cultural dimensions. HERON Laboratory, Computer Science Department. 2010. 
[15]. Brown, S. (2010). From VLEs to learning webs: the implications of Web 2.0 for learning and teaching. Interactive Learning Environments. Vol. 18, No. 1, 1-10.

[16]. Hao. Y \& Borich, G. A Practical Guide to Evaluate Quality of Online Courses. In Holim Song \& Terry Kidd. Handbook of Research on Human Performance and Instructional Technology. New York: Information science reference. 2010.

[17]. Hixon, E., Barczyk, C., Buckenmeyer, J., \& Feldman, L. Mentoring university faculty to become high quality online educators: A program evaluation. Online Journal of Distance Learning Administration, 2011, 14 (5).

[18]. Kaplan, A, M and Haenlein, M. Higher education and the digital revolution: About MOOCs, SPOCs, social media, and the Cookie Monster. Business horizon, 2016, 59, 441- 450.

[19]. Kazemifard, M; Ghasem- Aghaee, N and Oren, T. Emotive and cognitive simulation by agents: roles of three levels of information processing. Cognitive systems research, 2011, 13, 24-38.

[20]. Rocker, C; Janse, M; Portolm, N and Streitz, N. User requirements for intelligent home environments: A scenariodriven approach and empirical cross-cultural study. Published in: proceeding of the 2014 joint conference on intelligent objects and ambient intelligence, 2014, 111-116.

[21]. Mazadi, Z; Ghasem Aghaee, N and Oren, T. Prelude to cultural software agents: cultural backgrounds in agent simulation. Conference: Proceedings of the Spring Simulation Multi conference. 2008, 135-142.

[22]. Creswell J. w. Educational research: planning, conducting and evaluating quantitative and qualitative research. (Fourth edition). Pearson. Boston. 2012.

Heshmat Moradhaseli received the B.S. Degree in Computer Engineering from Payame Noor University, llam Center, Iran in 2011 and Master of Information Technology Management Degree from Azad University, electronici Tehran Branch, Iran in 2015. He is currently a PhD student at Azad University, Central Tehran Branch, Tehran, Iran. His research interest is in the development of intelligent technologies.

Jalal Haghighat Monfared is an assistant professor of Industrial Management at Azad University, Central Tehran Branch, Iran. His research is interest is in the development of intelligent technologies. 